\title{
Investigación de mercado de la crema de sábila (Aloe vera) de Aromas y Sabores del Desierto, Delicias, Chihuahua
}

Marketing research of sábila (aloe vera) cream of Aromas y Sabores del Desierto, Delicias, Chihuahua

\author{
Magaña-Magaña José Eduardo ${ }^{1 凶}$, Yadira Esmeralda Ramos-Lucero² ${ }^{2}$ Víctor Hugo Villarreal- \\ Ramirez $^{3}$, Manuel Soto-Zapata ${ }^{4}$ y Lorena Licón-Trillo ${ }^{5}$ \\ ${ }^{1}$ Facultad de Ciencias Agrícolas y Forestales de la Universidad Autónoma de Chihuahua \\ ${ }^{2}$ Posgrado de Agronegocios. ${ }^{3,4,5}$ Facultad de Ciencias Agrícolas y Forestales de la Universidad \\ Autónoma de Chihuahua.. \\ ${ }^{凶}$ Autor para correspondencia: emagana@uach.mx
}

Recibido: 11/01/2014

Aceptado: 14/07/2014

\section{RESUMEN}

El presente proyecto consistió en el desarrollo de una investigación de mercado del producto crema de sábila de la empresa aromas y sabores del desierto, la cual se encarga de la elaboración artesanal y comercialización del producto bajo el contexto de una microempresa. La empresa necesita saber si el mercado es suficientemente atractivo para ampliar su producción. El objetivo de esta investigación fue medir la atractividad del mercado de las personas de piel delicada o seca, diabéticos y a quienes les gusta las cremas naturales, identificar los gustos y preferencias de los consumidores y definir los canales de distribución. La población objetivo fueron las amas de casa en ciudad Delicias, Chihuahua. Esta encuesta se llevó a cabo aplicando un cuestionario como instrumento de recopilación de datos, el cual consto de 21 preguntas, y se aplicaron cara a cara. El tamaño de la muestra fue de 170 amas de casa. Los principales resultados nos indican que el $90.59 \%$ usa crema, la demanda de crema es en un 42.94\% para piel seca, $42.35 \%$ para la normal, $13.53 \%$ extraseca y el $.59 \%$ tanto para piel diabética y como para la grasosa, el consumo es de una hasta 4 cremas mensuales, el tipo de envase que prefieren es el tarro chico (100 grs) y el mediano (200 grs), el grado de aceptación de la crema de sábila es bueno, por lo que se puede concluir que si existe un mercado potencial.

Palabras claves: investigación de mercado, crema de sábila, encuesta. 


\begin{abstract}
This project was a market research of an aloe cream company named Aromas y Sabores del Desierto located in Delicias, Chihuahua, which is responsible for producing this product. The company tried to know if its product had a market attractive to expand its production. The objective of this research was to measure the attractiveness of the market for people sensitive or dry skin, diabetes and who like natural creams, identify the tastes and preferences of consumers and distribution channels defined. The target population was the housewives in town Delicias, Chihuahua. This survey was conducted using a questionnaire as a data collection instrument, which I consist of 21 questions, and applied face to face. The sample size was 170 housewives. The main results indicate that $90.59 \%$ use cream, cream demand is on a dry skin $42.94 \%, 42.35 \%$ for normal, $13.53 \%$ and $0.59 \%$ extra dry both diabetic skin and for oily, the consumption is from one to four monthly creams, the type of packaging they prefer is the guy jar (100 g) and medium (200 g), the degree of acceptance of the aloe vera cream is good, so we can conclude that if there is a potential market.
\end{abstract}

Keywords: market research, aloe cream, survey.

\section{INTRODUCCIÓN}

El consumo de productos compatibles con el medio ambiente, la salud y el desarrollo sustentable está aumentando a nivel mundial. Se estima que alrededor de 80 millones de personas han adoptado formas de consumo en esta línea al año 2010, concentrándose principalmente en EEUU y Europa.

Se denomina "Cosmética Natural" a aquella que basa sus formulaciones en ingredientes no sintéticos, representando estos al menos el $90 \%$ de sus componentes, considerándose innecesaria la adición de otro tipo de compuestos artificiales para lograr la efectividad de sus productos. Su premisa es cuidar y embellecer la piel en base a la utilización de materias existentes en la naturaleza.
Una de las materias primas de gran demanda en la cosmética natural es el aloe vera. La planta de Aloe vera es originaria de África, específicamente de la península de Arabia. Se ha utilizado durante siglos para la salud y la

belleza destacándose sus propiedades dermatológicas. La palabra Aloe deriva del árabe "alloeh" y significa "sustancia amarga brillante", mientras que "vera" significa verdad. Se le denomina también con el nombre de sábila.

La sábila tiene muchos beneficios en la piel, lo más destacable es su poder de penetración en las tres capas de la piel; dermis, epidermis e hipodermis, esto se debe a una sustancia que lleva el Aloe vera, la lignina, es una de las propiedades cosméticas más apreciadas, también expulsa al exterior las bacterias y los depósitos de grasa que taponan los poros de la piel. Al mismo tiempo, la acción

\title{
Revista Científica Biológico Agropecuaria Tuxpan 2 (1) ISSN: 2007-6940
}


de sus nutrientes naturales, las vitaminas, los minerales, los aminoácidos y las enzimas, estimulan la fabricación de nuevas células.

Por sus beneficios y poder de penetración en la piel conduce a que sea utilizado como base para cremas dirigidas para personas con piel débil o seca, así como también para piel diabética, las cuales han aumentado significativamente.

El uso de la crema es fundamental para ayudar a que la piel se conserve bella y saludable ya que evitan que la epidermis pierda humedad, la protegen de las agresiones ambientales y mejoran su apariencia. Ahora bien, es importante saber que la piel del cuerpo sufre proceso de envejecimiento, el cual es distinto al del rostro porque no se arruga con facilidad, pero sí pierde elasticidad, suavidad y humedad. Por esta razón, aplicar diariamente crema debe convertirse en hábito, pues ello no sólo contrarrestra la resequedad, sino que además restablece la película protectora de la epidermis que, inevitablemente, se deteriora durante el baño diario.

Aromas y sabores del desierto es una microempresa familiar creada hace 2 años, la cual se dedica a la fabricación artesanal de cremas de sábila, la materia prima que utiliza como base es el aloe vera o sábila $100 \%$ natural. Actualmente tiene poca distribución y desea saber si el mercado es suficientemente atractivo para ampliar su producción, por lo que se va a llevar a cabo un estudio de mercados.

La investigación de mercados es una técnica que utilizan cada vez más las empresas $\mathrm{y}$, en general, todo tipo de organizaciones ya que su función más importante es identificar las características de la demanda y del mercado, así como las necesidades y los gustos que imperan entre las personas.

Esta investigación tiene como objetivo principal analizar la demanda de la crema de sábila en ciudad Delicias, para poder medir la atractividad del mercado en las personas de piel delicada, diabéticos y a quienes les gusta las cremas naturales; identificar los gustos y preferencias de los consumidores; y definir los canales de distribución en ciudad Delicias. Analizar la demanda de la crema de sábila en ciudad Delicias, con el fin de ofrecer al consumidor un producto de excelente calidad elaborado a base de un producto natural.

\section{METODOLOGIA}

La investigación de mercados se desarrolló basándose en el modelo de Naresh K. Malhotra, 2008 (Fig. 1).

\section{Revista Científica Biológico Agropecuaria Tuxpan 2 (1) ISSN: 2007-6940}




\section{Definicion del problema}

\section{Desarrollo \\ del enfoque del problema}

\section{Diseño de}

investigacion

\section{Elaboracion y \\ presentacion del informe}
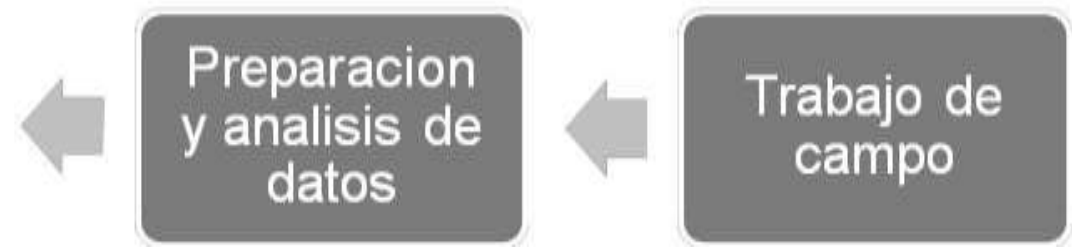

Figura 1. Proceso de investigación de mercados.

Algunas de las variables que se tomaron en cuenta fueron el nivel de ingresos, grado de aceptación, precio, preferencias del consumidor, hábitos de compra, consumo mensual.

Para obtener la información se aplicó la encuesta a una muestra de la población de ciudad Delicias, siendo la población objetivo las amas de casa ya que por lo general son ellas las que realizan las compras de la familia. Esta encuesta se llevó a cabo aplicando un cuestionario como instrumento de recopilación de datos, la entrevista se realizó personalmente.

Se determinó la muestra utilizando la fórmula de intervalos de confianza, la cual se basa en la construcción de intervalos de confianza alrededor de medias o proporciones de la muestra.
Se utilizaron dos variables criticas; una de ingresos y la otra la cantidad de consumo.

Variable crítica: cantidad de consumo.

Se aplicaron 15 pruebas pilotos las cuales se obtuvieron los siguientes resultados.

Media 1.73

Desviación típica 799

Varianza .638

Fórmula utilizada

$$
\mathbf{n}=\frac{\mathbf{Z}^{2} \mathbf{r}^{2}}{\mathbf{e}^{2}}
$$

$\mathrm{Z} 95 \%=1.96$

$\mathrm{r}^{2}=0.638$

$\mathrm{e}=7 \%$ de la media $=.1211$

$\mathrm{N}=\underline{(1.96)^{2}(.638)}$ 
$\mathrm{n}=170$

Se llevó a cabo un mapeo territorial de la zona y en base a la densidad de la población se consideró los siguientes porcentajes por sector para la aplicación de los cuestionarios.

Cuadro 1. Porcentajes por sector para la aplicación de cuestionarios.

\begin{tabular}{ccc}
\hline Sector & Porcentaje & Cuestionarios aplicados \\
\hline Norte & $15 \%$ & 25 \\
Sur & $30 \%$ & 52 \\
Oriente & $40 \%$ & 68 \\
Poniente & $15 \%$ & 25 \\
Total & $100 \%$ & 170 \\
\hline
\end{tabular}

Se elaboró una base de datos en el analizaron mediante gráficos, tablas de paquete estadístico SPSS; los datos se frecuencias, tablas de cruzadas. 


\section{RESULTADOS}

Cuadro 2. Tabla de contingencia 1.

Tabla de contingencia La crema que utiliza es para piel $*$ Con que humecta su pie

\begin{tabular}{|c|c|c|c|c|c|c|}
\hline \multirow[b]{3}{*}{ Normal } & \multirow[b]{3}{*}{ Recuento } & \multicolumn{3}{|c|}{ Con que humecta su piel } & \multirow[b]{2}{*}{ Aceite de } & \multirow[b]{2}{*}{ Total } \\
\hline & & crema & vaselina & ambas & & \\
\hline & & 65 & 1 & 5 & $\begin{array}{c}\text { almendras } \\
1\end{array}$ & 72 \\
\hline \multirow[b]{4}{*}{ Seca } & \% dentro de La crema que utiliza es para piel & $90.30 \%$ & $1.40 \%$ & $6.90 \%$ & $1.40 \%$ & $100.00 \%$ \\
\hline & $\%$ dentro de Con que humecta su piel & $42.20 \%$ & $100.00 \%$ & $35.70 \%$ & $100.00 \%$ & $42.40 \%$ \\
\hline & $\%$ del total & $38.20 \%$ & $0.60 \%$ & $2.90 \%$ & $0.60 \%$ & $42.40 \%$ \\
\hline & Recuento & 69 & 0 & 4 & 0 & 73 \\
\hline \multirow[b]{4}{*}{ Extra seca } & \% dentro de La crema que utiliza es para piel & $94.50 \%$ & $0.00 \%$ & $5.50 \%$ & $0.00 \%$ & $100.00 \%$ \\
\hline & $\%$ dentro de Con que humecta su piel & $44.80 \%$ & $0.00 \%$ & $28.60 \%$ & $0.00 \%$ & $42.90 \%$ \\
\hline & $\%$ del total & $40.60 \%$ & $0.00 \%$ & $2.40 \%$ & $0.00 \%$ & $42.90 \%$ \\
\hline & Recuento & 18 & 0 & 5 & 0 & 23 \\
\hline \multirow[b]{4}{*}{ Diabetica } & \% dentro de La crema que utiliza es para piel & $78.30 \%$ & $0.00 \%$ & $21.70 \%$ & $0.00 \%$ & $100.00 \%$ \\
\hline & $\%$ dentro de Con que humecta su piel & $11.70 \%$ & $0.00 \%$ & $35.70 \%$ & $0.00 \%$ & $13.50 \%$ \\
\hline & $\%$ del total & $10.60 \%$ & $0.00 \%$ & $2.90 \%$ & $0.00 \%$ & $13.50 \%$ \\
\hline & Recuento & 1 & 0 & 0 & 0 & 1 \\
\hline \multirow[b]{4}{*}{ Grasosa } & \% dentro de La crema que utiliza es para piel & $100.00 \%$ & $0.00 \%$ & $0.00 \%$ & $0.00 \%$ & $100.00 \%$ \\
\hline & $\%$ dentro de Con que humecta su piel & $0.60 \%$ & $0.00 \%$ & $0.00 \%$ & $0.00 \%$ & $0.60 \%$ \\
\hline & $\%$ del total & $0.60 \%$ & $0.00 \%$ & $0.00 \%$ & $0.00 \%$ & $0.60 \%$ \\
\hline & Recuento & 1 & 0 & 0 & 0 & 1 \\
\hline \multirow{7}{*}{ Total } & $\%$ dentro de La crema que utiliza es para piel & $100.00 \%$ & $0.00 \%$ & $0.00 \%$ & $0.00 \%$ & $100.00 \%$ \\
\hline & $\%$ dentro de Con que humecta su piel & $0.60 \%$ & $0.00 \%$ & $0.00 \%$ & $0.00 \%$ & $0.60 \%$ \\
\hline & $\%$ del total & $0.60 \%$ & $0.00 \%$ & $0.00 \%$ & $0.00 \%$ & $0.60 \%$ \\
\hline & Recuento & 154 & 1 & 14 & 1 & 170 \\
\hline & \% dentro de La crema que utiliza es para piel & $90.60 \%$ & $0.60 \%$ & $8.20 \%$ & $0.60 \%$ & $100.00 \%$ \\
\hline & $\%$ dentro de Con que humecta su piel & $100.00 \%$ & $100.00 \%$ & $100.00 \%$ & $100.00 \%$ & $100.00 \%$ \\
\hline & $\%$ del total & $90.60 \%$ & $0.60 \%$ & $8.20 \%$ & $0.60 \%$ & $100.00 \%$ \\
\hline
\end{tabular}

Como se observa en el cuadro anterior las personas encuestadas utilizan la crema, vaselina, ambas o el aceite de almendras para humectar su piel.

El $90.3 \%$ de piel normal humectan su piel con crema, el $1.4 \%$ con vaselina, el $6.9 \%$ con ambas y el $1.4 \%$ con aceite de almendras.
El $94.5 \%$ que compra para piel seca humecta su piel con crema, el 5.5\% con ambas.

El $78.3 \%$ que compra para piel extraseca humecta su piel con crema y el $21.7 \%$ humecta su piel con ambas.

Para piel diabética y piel grasosa el $.60 \%$ humectan su piel con crema. 
Cuadro 3. Tabla de contingencia 2.

\begin{tabular}{|c|c|c|c|c|c|}
\hline & Tabla de contingencia Que presentacion c & raria * Le inter & esaria comprar 1 & crema de sabila & \\
\hline & & Le interesaria & comprar la crem & de sabila & \\
\hline & & $\begin{array}{c}\begin{array}{c}\text { Me interesaria } \\
\text { mucho }\end{array} \\
\end{array}$ & $\begin{array}{c}\text { Me interesaria } \\
\text { regular }\end{array}$ & $\begin{array}{c}\text { Me interesaria } \\
\text { poco }\end{array}$ & Total \\
\hline Chica (100 grs) & Recuento & 22 & 22 & 18 & 62 \\
\hline & $\%$ dentro de Que presentacion compraria & $35.50 \%$ & $35.50 \%$ & $29.00 \%$ & $100.00 \%$ \\
\hline & $\%$ dentro de Le interesaria comprar la crema & $32.40 \%$ & $32.40 \%$ & $52.90 \%$ & $36.50 \%$ \\
\hline & $\%$ del total & $12.90 \%$ & $12.90 \%$ & $10.60 \%$ & $36.50 \%$ \\
\hline Mediana (200 g & s) Recuento & 26 & 33 & 8 & 67 \\
\hline & $\%$ dentro de Que presentacion compraria & $38.80 \%$ & $49.30 \%$ & $11.90 \%$ & $100.00 \%$ \\
\hline & $\%$ dentro de Le interesaria comprar la crema & $38.20 \%$ & $48.50 \%$ & $23.50 \%$ & $39.40 \%$ \\
\hline & $\%$ del total & $15.30 \%$ & $19.40 \%$ & $4.70 \%$ & $39.40 \%$ \\
\hline Grande (450 grs) & Recuento & 15 & 11 & 3 & 29 \\
\hline & $\%$ dentro de Que presentacion compraria & $51.70 \%$ & $37.90 \%$ & $10.30 \%$ & $100.00 \%$ \\
\hline & $\%$ dentro de Le interesaria comprar la crema & $22.10 \%$ & $16.20 \%$ & $8.80 \%$ & $17.10 \%$ \\
\hline & $\%$ del total & $8.80 \%$ & $6.50 \%$ & $1.80 \%$ & $17.10 \%$ \\
\hline Familiar (1000 & rs) Recuento & 5 & 2 & 5 & 12 \\
\hline & $\%$ dentro de Que presentacion compraria & $41.70 \%$ & $16.70 \%$ & $41.70 \%$ & $100.00 \%$ \\
\hline & $\%$ dentro de Le interesaria comprar la crema & $7.40 \%$ & $2.90 \%$ & $14.70 \%$ & $7.10 \%$ \\
\hline & $\%$ del total & $2.90 \%$ & $1.20 \%$ & $2.90 \%$ & $7.10 \%$ \\
\hline Total & Recuento & 68 & 68 & 34 & 170 \\
\hline & $\%$ dentro de Que presentacion compraria & $40.00 \%$ & $40.00 \%$ & $20.00 \%$ & $100.00 \%$ \\
\hline & $\%$ dentro de Le interesaria comprar la crema & $100.00 \%$ & $100.00 \%$ & $100.00 \%$ & $100.00 \%$ \\
\hline & $\%$ del total & $40.00 \%$ & $40.00 \%$ & $20.00 \%$ & $100.00 \%$ \\
\hline
\end{tabular}

Como se observa en el cuadro anterior las presentaciones de la crema son en tarros chicos de 100 grs, medianos de 200 grs, grandes de 450 grs, y la familiar de 1000 grs.

Al evaluar el interés de compra de los encuestados la presentación mediana es la que tiene mayor interés de compra con un $38 \%$ de gran interés y un $48.5 \%$ de interés regular, en segundo lugar la presentación chica de 100 grs tiene un $32.4 \%$ en el gran interés y un $32.4 \%$ en el regular interés en tanto que las otras presentaciones grande de 450 grs tienen un $22.10 \%$ y $16.20 \%$ respectivamente y la familiar un $7.4 \%$ y $2.9 \%$ respectivamente.

Finalmente los consumidores potenciales de gran interés hacia la crema de sábila representan un $40 \%$ y aquellos con interés regular también representan un $40 \%$. 
Cuadro 4. Tabla de contingencia 3.

\begin{tabular}{|c|c|c|c|c|c|c|c|}
\hline \multicolumn{8}{|c|}{ Tabla de contingencia Le gustan las cremas de productos naturales * Aproximadamente cuantas cremas compra mensualmente } \\
\hline & & \multicolumn{6}{|c|}{ Aproximadamente cuantas cremas compra mensualmente } \\
\hline & & 1 & 2 & 3 & 4 & Mas de 5 & Total \\
\hline \multirow[t]{4}{*}{ Me gustan mucho } & Recuento & 33 & 19 & 3 & 7 & 0 & 62 \\
\hline & $\%$ dentro de Le gustan las cremas de productos naturales & $53.20 \%$ & $30.60 \%$ & $4.80 \%$ & $11.30 \%$ & $0.00 \%$ & $100.00 \%$ \\
\hline & $\%$ dentro de Aproximadamente cuantas cremas compra mensualmente & $37.50 \%$ & $33.90 \%$ & $100.00 \%$ & $31.80 \%$ & $0.00 \%$ & $36.50 \%$ \\
\hline & $\%$ del total & $19.40 \%$ & $11.20 \%$ & $1.80 \%$ & $4.10 \%$ & $0.00 \%$ & $36.50 \%$ \\
\hline \multirow[t]{4}{*}{ Me gusta } & Recuento & 29 & 27 & 0 & 9 & 0 & 65 \\
\hline & $\%$ dentro de Le gustan las cremas de productos naturales & $44.60 \%$ & $41.50 \%$ & $0.00 \%$ & $13.80 \%$ & $0.00 \%$ & $100.00 \%$ \\
\hline & $\%$ dentro de Aproximadamente cuantas cremas compra mensualmente & $33.00 \%$ & $48.20 \%$ & $0.00 \%$ & $40.90 \%$ & $0.00 \%$ & $38.20 \%$ \\
\hline & $\%$ del total & $17.10 \%$ & $15.90 \%$ & $0.00 \%$ & $5.30 \%$ & $0.00 \%$ & $38.20 \%$ \\
\hline \multirow[t]{4}{*}{ Me gusta regular } & Recuento & 9 & 3 & 0 & 1 & 0 & 13 \\
\hline & $\%$ dentro de Le gustan las cremas de productos naturales & $69.20 \%$ & $23.10 \%$ & $0.00 \%$ & $7.70 \%$ & $0.00 \%$ & $100.00 \%$ \\
\hline & $\%$ dentro de Aproximadamente cuantas cremas compra mensualmente & $10.20 \%$ & $5.40 \%$ & $0.00 \%$ & $4.50 \%$ & $0.00 \%$ & $7.60 \%$ \\
\hline & $\%$ del total & $5.30 \%$ & $1.80 \%$ & $0.00 \%$ & $0.60 \%$ & $0.00 \%$ & $7.60 \%$ \\
\hline \multirow[t]{4}{*}{ Me gusta poco } & Recuento & 3 & 2 & 0 & 1 & 0 & 6 \\
\hline & $\%$ dentro de Le gustan las cremas de productos naturales & $50.00 \%$ & $33.30 \%$ & $0.00 \%$ & $16.70 \%$ & $0.00 \%$ & $100.00 \%$ \\
\hline & $\%$ dentro de Aproximadamente cuantas cremas compra mensualmente & $3.40 \%$ & $3.60 \%$ & $0.00 \%$ & $4.50 \%$ & $0.00 \%$ & $3.50 \%$ \\
\hline & $\%$ del total & $1.80 \%$ & $1.20 \%$ & $0.00 \%$ & $0.60 \%$ & $0.00 \%$ & $3.50 \%$ \\
\hline \multirow[t]{4}{*}{ No me gusta } & Recuento & 4 & 2 & 0 & 3 & 0 & 9 \\
\hline & $\%$ dentro de Le gustan las cremas de productos naturales & $44.40 \%$ & $22.20 \%$ & $0.00 \%$ & $33.30 \%$ & $0.00 \%$ & $100.00 \%$ \\
\hline & $\%$ dentro de Aproximadamente cuantas cremas compra mensualmente & $4.50 \%$ & $3.60 \%$ & $0.00 \%$ & $13.60 \%$ & $0.00 \%$ & $5.30 \%$ \\
\hline & $\%$ del total & $2.40 \%$ & $1.20 \%$ & $0.00 \%$ & $1.80 \%$ & $0.00 \%$ & $5.30 \%$ \\
\hline \multirow[t]{4}{*}{ No tengo informacion } & Recuento & 10 & 3 & 0 & 1 & 1 & 15 \\
\hline & $\%$ dentro de Le gustan las cremas de productos naturales & $66.70 \%$ & $20.00 \%$ & $0.00 \%$ & $6.70 \%$ & $6.70 \%$ & $100.00 \%$ \\
\hline & $\%$ dentro de Aproximadamente cuantas cremas compra mensualmente & $11.40 \%$ & $5.40 \%$ & $0.00 \%$ & $4.50 \%$ & $100.00 \%$ & $8.80 \%$ \\
\hline & $\%$ del total & $5.90 \%$ & $1.80 \%$ & $0.00 \%$ & $0.60 \%$ & $0.60 \%$ & $8.80 \%$ \\
\hline \multirow[t]{4}{*}{ Total } & Recuento & 88 & 56 & 3 & 22 & 1 & 170 \\
\hline & $\%$ dentro de Le gustan las cremas de productos naturales & $51.80 \%$ & $32.90 \%$ & $1.80 \%$ & $12.90 \%$ & $0.60 \%$ & $100.00 \%$ \\
\hline & $\%$ dentro de Aproximadamente cuantas cremas compra mensualmente & $100.00 \%$ & $100.00 \%$ & $100.00 \%$ & $100.00 \%$ & $100.00 \%$ & $100.00 \%$ \\
\hline & $\%$ del total & $51.80 \%$ & $32.90 \%$ & $1.80 \%$ & $12.90 \%$ & $0.60 \%$ & $100.00 \%$ \\
\hline
\end{tabular}

Las personas encuestadas mensualmente compran aproximadamente de 1 a más de 5 cremas.

Al evaluar el gusto por la cremas de productos naturales nos encontramos que a las personas encuestadas les gusta las cremas de productos naturales en un $44.6 \%$ y son aquellos que tienen una intención de compra de una crema al mes, el $41.5 \%$ compra 2 y el $13.8 \%$ compran 4.

En segundo lugar tenemos a los que les gustan mucho las cremas de productos naturales con un $53.2 \%$ que compran aproximadamente 1 crema al mes, un $30.6 \%$ a los que compran 2, un $4.8 \%$ a los de 3 y un $11.3 \%$ a los que compran 4.

En tercer lugar tenemos a los encuestados que no tienen información sobre las cremas de productos naturales, en cuarto lugar tenemos a los que les gusta regular, en quinto a los que nos les gusta y por último a los que les gusta poco. 
Cuadro 5. Tabla de contingencia 4.

\begin{tabular}{|c|c|c|c|c|c|c|}
\hline \multicolumn{7}{|c|}{ Tabla de contingencia Cuanto le gustaria pagar por ella * Que presentacion compraria } \\
\hline \multicolumn{7}{|c|}{ Que presentacion compraria } \\
\hline & & Chica (100 grs) & Mediana (200 grs) & Grande (450 grs) & Familiar (1000 grs) & Total \\
\hline \multirow[t]{4}{*}{$0-20$ pesos } & Recuento & 42 & 22 & 0 & 1 & 65 \\
\hline & $\%$ dentro de Cuanto le gustaria pagar por ella & $64.60 \%$ & $33.80 \%$ & $0.00 \%$ & $1.50 \%$ & $100.00 \%$ \\
\hline & $\%$ dentro de Que presentacion compraria & $67.70 \%$ & $32.80 \%$ & $0.00 \%$ & $8.30 \%$ & $38.20 \%$ \\
\hline & $\%$ del total & $24.70 \%$ & $12.90 \%$ & $0.00 \%$ & $0.60 \%$ & $38.20 \%$ \\
\hline \multirow[t]{4}{*}{ 20-40 pesos } & Recuento & 18 & 29 & 12 & 2 & 61 \\
\hline & $\%$ dentro de Cuanto le gustaria pagar por ella & $29.50 \%$ & $47.50 \%$ & $19.70 \%$ & $3.30 \%$ & $100.00 \%$ \\
\hline & $\%$ dentro de Que presentacion compraria & $29.00 \%$ & $43.30 \%$ & $41.40 \%$ & $16.70 \%$ & $35.90 \%$ \\
\hline & $\%$ del total & $10.60 \%$ & $17.10 \%$ & $7.10 \%$ & $1.20 \%$ & $35.90 \%$ \\
\hline \multirow[t]{4}{*}{ 40-60 pesos } & Recuento & 2 & 14 & 12 & 3 & 31 \\
\hline & $\%$ dentro de Cuanto le gustaria pagar por ella & $6.50 \%$ & $45.20 \%$ & $38.70 \%$ & $9.70 \%$ & $100.00 \%$ \\
\hline & $\%$ dentro de Que presentacion compraria & $3.20 \%$ & $20.90 \%$ & $41.40 \%$ & $25.00 \%$ & $18.20 \%$ \\
\hline & $\%$ del total & $1.20 \%$ & $8.20 \%$ & $7.10 \%$ & $1.80 \%$ & $18.20 \%$ \\
\hline \multirow[t]{4}{*}{60 o mas } & Recuento & 0 & 2 & 5 & 6 & 13 \\
\hline & $\%$ dentro de Cuanto le gustaria pagar por ella & $0.00 \%$ & $15.40 \%$ & $38.50 \%$ & $46.20 \%$ & $100.00 \%$ \\
\hline & $\%$ dentro de Que presentacion compraria & $0.00 \%$ & $3.00 \%$ & $17.20 \%$ & $50.00 \%$ & $7.60 \%$ \\
\hline & $\%$ del total & $0.00 \%$ & $1.20 \%$ & $2.90 \%$ & $3.50 \%$ & $7.60 \%$ \\
\hline \multirow[t]{4}{*}{ Total } & Recuento & 62 & 67 & 29 & 12 & 170 \\
\hline & $\%$ dentro de Cuanto le gustaria pagar por ella & $36.50 \%$ & $39.40 \%$ & $17.10 \%$ & $7.10 \%$ & $100.00 \%$ \\
\hline & $\%$ dentro de Que presentacion compraria & $100.00 \%$ & $100.00 \%$ & $100.00 \%$ & $100.00 \%$ & $100.00 \%$ \\
\hline & $\%$ del total & $36.50 \%$ & $39.40 \%$ & $17.10 \%$ & $7.10 \%$ & $100.00 \%$ \\
\hline
\end{tabular}

En el cuadro anterior se observa la cantidad en dinero que a los encuestados les gustaría pagar por la crema de su preferencia que va desde 0-20 pesos, de 20-40 pesos, 40-60 pesos y por ultimo de 60 o más.

Dentro del rango de 0-20 pesos un $67.7 \%$ compraría la crema chica, un $32.8 \%$ compraría la mediana y un $8.30 \%$ la grande.

De $20-40$ pesos, un $29 \%$ compraría la chica, un $43.3 \%$ la mediana, $41.4 \%$ la grande y un $16.7 \%$ la familiar. De $40-60$ pesos. El $3.20 \%$ compraría la chica, el $20.9 \%$ la mediana, el $41.4 \%$ la grande y un $25 \%$ la familiar. Por ultimo dentro del rango de 60 o más, un 3\% compraría la mediana, el $17.20 \%$ la grande y el $50 \%$ la familiar.
Podemos concluir que las presentaciones mediana y chica son la preferidas de los consumidores potenciales con un $39.40 \%$ y un $36.5 \%$ respectivamente.

\section{CONCLUSIONES}

Si existe un mercado potencial para la crema de sábila, esto se puede concluir ya que el $90.59 \%$ de los consumidores potenciales compran crema, les gusta las cremas naturales y les interesaría comprar la crema de sábila y existe demanda de crema para pieles delicadas o secas, para pieles normales, para los que les gustan las cremas naturales y para los diabéticos. 
Las presentaciones chica (100 grs) y mediana (200 grs) son las preferidas por los consumidores potenciales, los cuales están dispuestos a pagar de $0-20$ pesos y de $0-40$ pesos respectivamente.

Los consumidores potenciales prefieren comprar la crema de sábila en los abarrotes y en las tiendas de autoservicio.

Se recomienda que la empresa siga con el crecimiento y desarrollo de mercado.

\section{LITERATURA CITADA}

Mahotra, N. K. 2008. Investigación de mercados, editorial Prentice Hall, 5 edición.

Ben

Copyright (c) 2014 José Eduardo Magaña Magaña, Yadira Esmeralda Ramos Lucero, Victor Hugo Villarreal Trillo, Manvel Soto Zapata y http://www.monografia.com/trabajos10/aloe/alo e.shtml día 15 de febrero de 2013. Hora de consulta 17:45 p.m.

Gerencia de investigación de mercados y subgerencia de estadísticas (2011) Perfil económico Aloe Vera www.ceird.gov.do/estudios_economicos lestu.

http://www.elaloevera.com/ día 22 de mayo de

2013. Hora de consulta 22:10 p.m www.lohas.com día 2 de marzo de 2013. Hora de consulta 20:18 p.m.

Este texto está protegido por una licencia Creative Commorr 4.0 .

Usted es libre para Compartir —copiar y red istribuir el material en cualquier medio o formato-y Ad aptar el documento —remezclar, transformar y crear a partir del material- para cualquier propósito, incluso para fines comerciales, siempre que cumpla la condición de:

Atribución: Usted debe dar crédito a la obra original de manera adecuada, proporcionar un enlace a la licencia, e indicar si se han realizado cambios. Puede hacerlo en cualquier forma razonable, pero no de forma tal que sugiera que tiene el apoyo del licenciante olo recibe por el usoque hace de la obra.

Resumendelicencia - Textocompletodelalicenia 